\title{
UnTTrapping the ends: A new player in overcoming protein linked DNA damage
}

\author{
Karim Bahmed ${ }^{1}$, Karin C Nitiss ${ }^{1}$, John L Nitiss ${ }^{1}$ \\ ${ }^{\prime}$ Molecular Pharmacology Department, St. Jude Children's Research Hospital, 262 Danny Thomas Place, Memphis, TN 38105, USA \\ Cell Research (2010) 20:122-123. doi:10.1038/cr.2010.17; published online 1 February 2010
}

DNA topoisomerases carry out their reactions by generating transient covalent phosphotyrosine intermediates with DNA [1]. This reaction mechanism allows these enzymes that need to break DNA to do so in a way that does not induce genome rearrangements or DNA damage responses. However, a variety of agents are able to trap topoisomerases while they are covalently bound to DNA. These agents include small molecules that are active and important anti-cancer or anti-bacterial agents $[2,3]$. Trapping of topoisomerases leads to DNA damage that includes strand breaks, and protein covalently bound to DNA. While strand breaks can be repaired by conventional break repair pathways such as homologous recombination and non-homologous end-joining, the removal of protein covalently bound to DNA is an interesting and unusual challenge for cells.

There are two major classes of enzymatic activities that can repair topoisomerase-mediated DNA damage: nucleases and other phosphodiesterases. Nucleases cut DNA internal to a trapped topoisomerase, while phosphodiesterases specifically cleave the phosphotyrosine linkage initially formed during topoisomerase-mediated cleavage of DNA. Several nucleases

Correspondence: John L Nitiss Tel: +901-595-2794; Fax: +901-595-4290

E-mail: john.nitiss@stjude.org have been implicated in removing topoisomerases covalently bound to DNA, most importantly nucleases associated with the Mre11/Rad50/Nbs1 (MRN) complex. Specific nuclease deficient mutations in either Mre11 or Rad50 prevent the processing of Spo11:DNA complexes arising from Spo11. Spo11 is a type IIB topoisomerase homolog that introduces the DNA double strand breaks that initiate meiotic recombination [4]. S. cerevisiae sae2 mutants are also deficient in disjoining Spo11 from DNA, and work in S. pombe has demonstrated that the Sae2 homolog Ctp1 (CtIP in mammalian cells) can disjoin trapped Top2 from DNA [5].

In addition to nuclease removal of trapped topoisomerases, specific phosphodiesterases are also able to process trapped topoisomerases by cleavage of the enzyme:DNA phosphotyrosine linkage. The first identified enzyme that could carry out this reaction was identified in yeast and termed Tdp1 (tyrosyl DNA phosphodiesterase I) by Nash and colleagues [6, 7]. Subsequent work demonstrated that this protein was found in all eukaryotes, and showed that the enzyme was a phospholipase D superfamily enzyme $[8,9]$. While the initial characterization of the yeast enzyme suggested that Tdpl was specific for $3^{\prime}$ damage to DNA, and could disjoin Top1 trapped on DNA, subsequent studies demonstrated that yeast Tdp1 also protected cells from Top2-mediated DNA damage, and could remove phosphotyrosyl linked peptides from both the $5^{\prime}$ and $3^{\prime}$ ends of DNA [10]. Since the mammalian enzyme apparently lacks the ability to process $5^{\prime}$ phosphotyrosyl linked peptides [11], other mammalian enzymes might process 5 ' phosphotyrosyl "adducts". Work by Ledesma and colleagues has found an enzyme, TTRAP that has this property [12].

Ledesma et al. identified TTRAP in a screen that was designed to detect enzymes besides Tdp1 that could process $3^{\prime}$ phosphotyrosyl adducts. They screened a mammalian cDNA library in a yeast strain lacking both Tdp1 and Rad1. Rad1 is a homolog of mammalian XPF, and ERCC1/XPF is a heterodimer nuclease that participates in nucleotide excision repair, and in other DNA repair pathways. Genetic evidence suggested that ERCC1/XPF is a component of another pathway for processing Top1 damage; therefore the double mutant strain has substantial camptothecin sensitivity. The two genes identified in the screen were Tdp 1 and TTRAP. As expected from the genetic screen, purified TTRAP protein can process Top1-mediated DNA damage, although it processes 3' phosphotyrosyl moieties rather inefficiently. TTRAP is substantially more active with 5 ' phosphotyrosyl modified oligonucleotides as a substrate. Importantly, siRNA knockdown of TTRAP results in sensitivity to the Top2 targeting agent etoposide, but not to the Top 1 targeting agent 
camptothecin. Consistently, overexpression of TTRAP in yeast cells confers resistance to etoposide compared to wild type cells. These results strongly implicate TTRAP as an important activity for repairing Top2-mediated DNA damage [12].

Repair of either Top1- or Top2-mediated damage is a complicated biological process. The first step requires recognition that the enzyme is "trapped". This recognition probably relies on the interference of the trapped complex with ongoing DNA metabolic events such as replication or transcription. In dividing cells, recognition of trapped complexes is associated with replication fork collisions. For non-replicating cells, collision with transcribing RNA polymerase is likely to be important. After recognition of a trapped complex, an initial repair event may include proteasome-mediated degradation of the topoisomerase [13]. The proteolysis is critical for several reasons. First, once degradation of the trapped protein commences, the enzyme probably will be unable to reseal the topoisomerase induced break. Therefore, the initiation of proteolysis commits the cell to repair the topoisomerase mediated damage. Second, proteolysis cannot completely remove the protein. A phosphotyrosyl adduct will remain bound to DNA, requiring enzymes such as Tdp1 and TTRAP to completely remove the protein. Proteolysis is important since Tdp1 is relatively inactive against intact Top1 trapped on DNA. It will be interesting to determine whether the TTRAP requires proteolysis of trapped topoisomerases.

A major reason why Tdp1 attracted attention in addition to its unique enzymatic activity was the recognition that mutations in Tdp1 could lead to a human genetic disorder, SCAN1 (spinocerebellar ataxia with axonal neuropathy) [14]. Transgenic mouse models have provided support for the hypothesis that Tdp1 represents one of many DNA repair functions that are neuroprotective [15]. Since TTRAP was originally identified as a protein associated with TNF receptor family members, and plays an important role in Zebrafish embryogenesis [16, 17], complete lossof-function mutations may be difficult to study in mouse systems. Nonetheless, once they are better understood, the pathways that include TTRAP should illuminate the repair of topoisomerase mediated damage, and perhaps, lead to a better understanding of how DNA repair functions are required during neuronal development.

\section{References}

1 Nitiss JL. DNA topoisomerase II and its growing repertoire of biological functions. Nat Rev Cancer 2009; 9:327-337.

2 Nitiss JL. Targeting DNA topoisomerase II in cancer chemotherapy. Nat Rev Cancer 2009; 9:338-350.

3 Pommier Y, Barcelo JM, Rao VA et al. Repair of topoisomerase I-mediated DNA damage. Prog Nucleic Acid Res Mol Biol 2006; 81:179-229.

4 Keeney S, Giroux CN, Kleckner N. Meiosis-specific DNA double-strand breaks are catalyzed by Spo11, a member of a widely conserved protein family. Cell 1997; 88:375-384.

5 Hartsuiker E, Neale MJ, Carr AM. Distinct Requirements for the Rad32(Mre11) Nuclease and Ctp1(CtIP) in the Removal of Covalently Bound Topoisomerase I and II from DNA. Mol Cell 2009; 33:117123.

6 Pouliot JJ, Yao KC, Robertson CA, Nash HA. Yeast gene for a Tyr-DNA phosphodiesterase that repairs topoisomerase I complexes. Science 1999; 286:552-555.

7 Yang SW, Burgin AB, Jr., Huizenga $\mathrm{BN}$ et al. A eukaryotic enzyme that can disjoin dead-end covalent complexes between DNA and type I topoisomerases. Proc Natl Acad Sci USA
1996; 93:11534-11539.

8 Davies DR, Interthal H, Champoux JJ, Hol WG. Insights into substrate binding and catalytic mechanism of human tyrosyl-DNA phosphodiesterase (Tdp1) from vanadate and tungstate-inhibited structures. J Mol Biol 2002; 324:917-932.

9 Davies DR, Interthal H, Champoux JJ, Hol WG. The crystal structure of human tyrosyl-DNA phosphodiesterase, Tdp1. Structure 2002; 10:237-248.

10 Nitiss KC, Malik M, He X, White SW, Nitiss JL. Tyrosyl-DNA phosphodiesterase (Tdp1) participates in the repair of Top2mediated DNA damage. Proc Natl Acad Sci USA 2006; 103:8953-8958.

11 Interthal $\mathrm{H}$, Chen HJ, Champoux JJ. Human Tdp1 cleaves a broad spectrum of substrates, including phosphoamide linkages. J Biol Chem 2005; 280:3651836528.

12 Ledesma FC, El Khamisy SF, Zuma MC, Osborn K, Caldecott KW. A human 5'-tyrosyl DNA phosphodiesterase that repairs topoisomerase-mediated DNA damage. Nature 2009; 461:674-678.

13 Zhang AL, Lyu YL, Lin CP et al. A protease pathway for the repair of topoisomerase II-DNA covalent complexes. J Biol Chem 2006; 281:35997-36003.

14 Takashima H, Boerkoel CF, John J et al. Mutation of TDP1, encoding a topoisomerase I-dependent DNA damage repair enzyme, in spinocerebellar ataxia with axonal neuropathy. Nat Genet 2002; 32:267-272.

15 Katyal S, El-Khamisy SF, Russell HR et al. TDP1 facilitates chromosomal single-strand break repair in neurons and is neuroprotective in vivo. EMBO $J$ 2007; 26:4720-4731.

16 Esguerra CV, Nelles L, Vermeire L et al. Ttrap is an essential modulator of Smad3-dependent Nodal signaling during zebrafish gastrulation and left-right axis determination. Development 2007; 134:4381-4393.

17 Pype S, Declercq W, Ibrahimi A et al. TTRAP, a novel protein that associates with CD40, tumor necrosis factor (TNF) receptor-75 and TNF receptorassociated factors (TRAFs), and that inhibits nuclear factor- $\kappa \mathrm{B}$ activation. $J$ Biol Chem 2000; 275:18586-18593. 